\title{
Recent developments in spatial econometrics
}

\author{
Nikolaos Antonakakis ${ }^{1,2}$
}

Received: 18 November 2019 / Accepted: 11 December 2019 / Published online: 18 December 2019 (c) Springer-Verlag GmbH Germany, part of Springer Nature 2019

The papers appearing in this Special Issue of the Journal of Geographical Systems come from the XII World Conference of the Spatial Econometrics Association, held at Webster Vienna Private University in Vienna on June 11-12, 2018.

The first paper in this issue is by James LeSage and presents some new computational breakthroughs in MCMC estimation for a family of spatial regression models involving multiple simultaneous dependence parameters. This includes space-time dynamic panel models, origin-destination flow models and models involving convex combinations of spatial weight matrices. During estimation, the author samples from each of the conditional distributions of the model parameters using Metropolis-Hastings $(\mathrm{M}-\mathrm{H})$, then evaluates the joint posterior for each set of parameter draws. The $\mathrm{M}-\mathrm{H}$ samples of the parameters hit the areas of high-density support allowing an accurate Monte Carlo integration estimate of the log-marginal likelihood. The log-marginal likelihood is used to calculate posterior model probabilities that allow model comparison for different weight matrices, model specifications, etc. For a cross-sectional model involving 20,000 observations, 100,000 draws can be produced in around $1 \mathrm{~min}$ on a laptop computer. A space-time dynamic panel model involving one million observations ( $N$ times $T$ ) can be estimated in 20 min.

The focus of the second paper, by James LeSage and Manfred M. Fischer, is on cross-sectional dependence model specifications in a static trade panel data setting, and suggests alternative specifications for modeling time-invariant factors such as sociocultural indicator variables, e.g., common language and currency. These are typically treated as a source of heterogeneity that is eliminated using fixed effects transformations, but the authors find evidence of cross-sectional dependence after eliminating country-specific and time-specific effects. These findings suggest use of alternative simultaneous dependence model specifications that accommodate crosssectional dependence, which the authors set forth along Bayesian estimation methods. Ignoring cross-sectional dependence implies biased estimates from panel trade

Nikolaos Antonakakis

nikolaos.antonakakis@webster.ac.at

1 Business and Management Department, Webster Vienna Private University, Praterstraße 23, 1020 Vienna, Austria

2 Economics and Finance Group, Business School, University of Portsmouth, Portland Street, Portsmouth PO1 3DE, UK 
flow models that rely on fixed effects. The alternative cross-sectional specifications suggested are tested using panel data on trade flows covering 74 countries from 1963 to 2000. Another methodological innovation of this paper is use of cross-sectional dependence weight matrix structures. The weight matrix structures are constructed to reflect spatial proximity between countries, as well as numerous types of sociocultural proximity such as common currency, language, and colonial ties. A convex combination of these multiple weight structures allows the authors to treat sociocultural factors that have been traditionally modeled as time-invariant fixed effects, as sources of cross-sectional dependence.

In the third and final paper of the special issue, written by Bernard Fingleton, Italy's possible exit from the EU, or Italexit, and its implication for trade flows between Italian regions is assessed in terms of its impact on employment, using a time-space dynamic panel data model with a spatial moving average random effects structure of the disturbances. The paper is a companion paper to an earlier prediction exercise regarding Brexit. The model includes spatial and temporal dependencies involving the endogenous variable, leading to estimates based on a new dynamic spatial generalized moments estimator. The predictions use modified interregional trade estimates, assuming a reduction in trade flows between Italian and EU regions due to Italexit, to simulate the impact on employment across Italian regions and the wider EU. Comparisons are made between Italexit and Brexit.

Acknowledgements The guest editor would like to thank the editors of this journal as well as the referees of this special issue for their contribution.

Publisher's Note Springer Nature remains neutral with regard to jurisdictional claims in published maps and institutional affiliations. 